\title{
Autonomous boat dynamics: How far away is simulation from the high sea?
}

\author{
Ya Huang \\ School of Engineering \\ University of Portsmouth \\ Portsmouth, UK \\ ya.huang@port.ac.uk
}

\author{
Ze Ji \\ School of Engineering \\ Cardiff University \\ Cardiff, UK \\ jiz1@cardiff.ac.uk
}

\begin{abstract}
The study demonstrates the process of implementing a 3-degrees-of-freedom surge-sway-yaw boat dynamic model in a numeric simulation environment. Estimated environmental disturbance force introduced in the simulation provides a scope for determining boat thrust force range and thrust angle range. The basic simulation framework allows the designer of a small robotic boat to change control logics in relation to the actuator (thruster) layout without the construction of a prototype. The study draws on the key assumptions of hydrodynamic added masses and damping coefficients, and indicates ways to estimate these parameters. The framework offers a starting point for anyone working on mechanical design of a robotic test boat for developing any control algorithms.
\end{abstract}

Keywords-Autonomous surface vehicle; control; simulation.

\section{INTRODUCTION}

The study intends to develop a basic dynamic simulation platform to identify design parameters of unmanned surface vehicle (USV). The simulation would consider operational requirements, such as time to reach maximum forward speed, minium radius of turn, and tolerance behaviour near a waypoint. With altered boat mass, mass distribution, and location of thruster(s), these characteristics would change correspondingly. Disturbance forces from wind, wave and curent play a role on manoeuverability - so-called seakeeping in the maritime community. Taking a single vectored thruster boat model, for example, the first essential step of design is to identify the maximum thrust force and the range of yaw angles of its propulsion system, based on a comnbination of various factors. Thorough mathematical treatment of these factors exists [1, 2], but implementing changes to such a web of interlinked variables is cumbersome and may lead to unforeseen singularities. The present study serves as a first step for mechanical design of a test boat examining effects of the key variables on design parameters saving on prototyping.

The simulation is based on, but not limited to, a prototype of an unmanned catamaran of one-metre long (Fig. 1). The boat was built as a student group project of five mechanical and electronic engineering students at the University of Portsmouth in 2016*. Autonomous navigation was implemented on a Raspberry Pi 2 micro processor, enabling the boat to follow a list of predefined waypoints. The boat motion was regulated by a vectored motor (or 'azipod', thruster) - a servo steered motor. The original engineering challenge was to reach between two waypoints with wave current disturbances

* The student project at Portsmouth was sponsored by IBM Client Innovation Centre, Hursley UK as an aid to lifeboat crew. The highly variable wave current conditions in open water drove the team to change motor capacity and positions. The present study hopes to address such design consideration at an early stage.
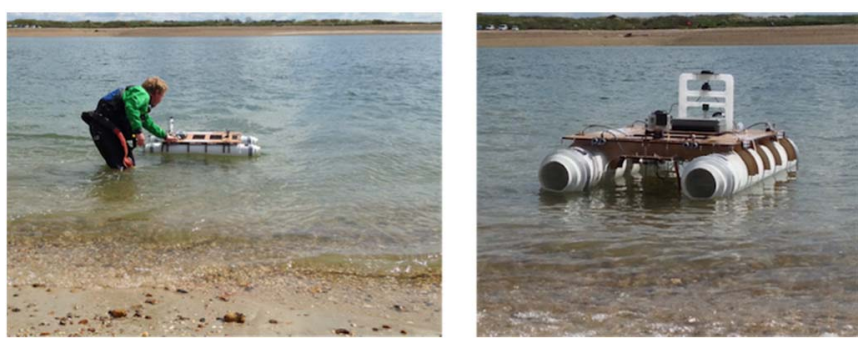

Fig. 1. A student retrieving the Pytheas unmanned boat after completing 3 return journeys crossing the 400-m wide open seawater between Southsea and Hayling Island within 10-m diameter of the predefined GPS coordinates (waypoints) based on the online map service by Google ${ }^{\circledR}$.

The parametric study of boat dynamics will help set operational envelops for applying any control strategies at close proximity, be it collision avoidance or path planning. Knowledge of vehicle characteristics, such as linear and rotational inertia and propulsion-weight ratio, may improve the implementation of the Convention on the International Regulations for Preventing Collisions at Sea (COLREGs). Such practice is not widely observed in the USV arena [2].

Regarding collision avoidance, the majority of previous works of control have been focused on adopting solutions from mobile robotics directly to USVs, such as A* path planning or its descendent [2]. But inadequate attention was paid to the challenge of vehicle control in high sea states [3]. At immediate close proximity of obstructing waves, any planning strategy would rely on the relative strength of boat propulsion system to environtmental disturbance forces. The simulation intends to associate mechanical design with control logics.

Estimates of boat hydrodynamic characteristics inevitably affect boat dynamic simulation resutls. One challenge of the numerical modelling effort has been to identify the hydrodynamic added masses and damping coefficients, when considering boat surge, sway and yaw $[4,5]$. The motivation is to implement a three degrees of freedom rigid body dynamic model of the boat without exhaustive hydrodyanmic and computational fluidic dynamic realisations. The MATLABSimulink ${ }^{\circledR}$ package is used to demonstrate the parametric study in a tutorial style. 


\section{METHOD}

\section{A. Kinematics and kinetics of boat model}

The dynamic boat model consideres 2-Dimensional plane motion with three degrees of freedom (3DOF) in the bodyfixed coordinate frame of $\mathrm{x}$ (surge) and $\mathrm{y}$ (sway) for translation, and $\mathrm{z}$ (yaw) for rotation of the boat body in the global inertia frame of XYZ.

The propulsion system comprised a servo steered motor producing a thrust force $F$ and a thrust angle $b$ (Fig. 2).

It is assumed that disturbances from a combination of wave, current and wind could be represented by a force $F_{\mathrm{d}}$ as a function of time applied at angle $k$ in the three equations of motion (Fig. 2).

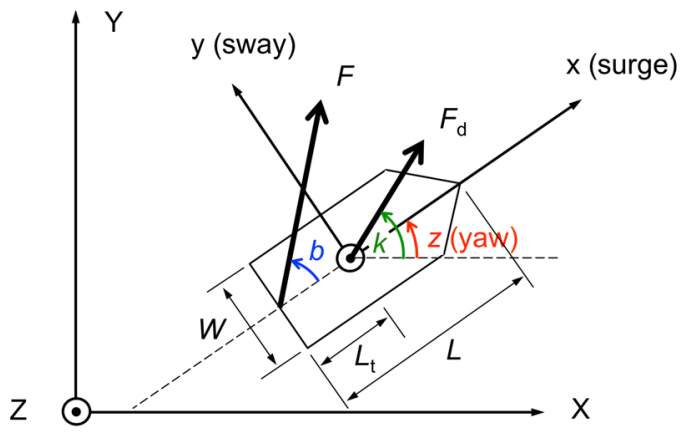

Fig. 2. Free body diagram of boat in the body-fixed (xyz) and global inertial frame (XYZ) with main forces and dimensions. $F_{\mathrm{d}}$ is exerted at boat mass centre. See Tables I and II for symbols.

With reference to Fig. 2, the kinematic relationship between the boat position in the global inertial frame XYZ and the boat body-fixed frame xyz can be defined.

$$
\begin{aligned}
& {[X]=[x] \cos [z]-[y] \sin [z]} \\
& {[Y]=[y] \cos [z]+[x] \sin [z]} \\
& {[Z]=[z]}
\end{aligned}
$$

where $[X]$ and $[Y]$ represent translational position $(X, Y)$, velocity $\left(X^{\prime}, Y^{\prime}\right)$ or acceleration $\left(X^{\prime \prime}, Y^{\prime \prime}\right)$ in the global inertial frame; $[x]$ and $[y]$ denote translational position $(x, y)$, velocity $\left(x^{\prime}, y^{\prime}\right)$ or acceleration $\left(x^{\prime \prime}, y^{\prime \prime}\right)$ in the local body-fixed frame attached to mass centre of the boat; $[z]$ comprises the yaw angle $(z)$, yaw angular velocity $\left(z^{\prime}\right)$, or yaw angular acceleration ( $z$ "). $[Z]$ and $[z]$ both measure yaw and are identical in both reference frames, and therefore $z, z$ ' and $z$ " are used throughout to express any rotation or yaw.

Using the general Newton's Second Law $\sum F=m a$ and $\sum M=I \alpha$, where $\sum F$ and $\sum M$ are external forces and moments and $a$ and $\alpha$ are linear and angular accelerations, the equations of motion expressed in the local xyz frame take the form $[1,3]$ :

$$
\begin{aligned}
& F \cdot \cos (b)+F_{\mathrm{d}} \cdot \cos (k-z)+\left(m+m_{\mathrm{yy}}\right) \cdot y^{\prime} \cdot z^{\prime}-d_{1} \cdot \operatorname{sign}\left(x^{\prime}\right) \cdot /\left.x^{\prime}\right|_{1} \\
& =\left(m+m_{\mathrm{xx}}\right) \cdot x^{\prime \prime} \\
& F \cdot \sin (b)+F_{\mathrm{d}} \cdot \sin (k-z)-\left(m+m_{\mathrm{xx}}\right) \cdot x^{\prime} \cdot z^{\prime}-d_{2} \cdot \operatorname{sign}\left(y^{\prime}\right) \cdot / y^{\prime} / a_{2} \\
& =\left(m+m_{\mathrm{yy}}\right) \cdot y^{\prime \prime}
\end{aligned}
$$

$L_{\mathrm{t}} \cdot F \cdot \sin (b)-\left(m_{\mathrm{yy}}-m_{\mathrm{xx}}\right) \cdot x^{\prime} \cdot y^{\prime}-d_{3} \cdot \operatorname{sign}(z) \cdot / z^{\prime} /^{a_{3}}$

$=\left(I+I_{\mathrm{zz}}\right) \cdot Z^{\prime \prime}$

The geometric and inertia parameters in the above equations are summarised in Table I. These are based on a

\begin{tabular}{|c|c|c|}
\hline & Value & Defintion \\
\hline$L$ & $1 \mathrm{~m}$ & Overall length of boat \\
\hline$L_{\mathrm{t}}$ & $0.4 \mathrm{~m}$ & $\begin{array}{l}\text { Distance between mass centre and point } \\
\text { of action of thrust force }\end{array}$ \\
\hline$W$ & $0.55 \mathrm{~m}$ & Width of boat \\
\hline$m$ & $7 \mathrm{~kg}$ & Overall mass of boat \\
\hline$m_{\mathrm{xx}}$ & $(r m) \mathrm{kg}$ & $\begin{array}{l}\text { Added mass in the surge } \mathrm{X} \text {-axis of boat } \\
{[4,5], r=[0.01,0.05]}\end{array}$ \\
\hline$m_{\mathrm{yy}}$ & $\left(p \pi \mathrm{D}^{2} \mathrm{~L} / 2\right) \mathrm{kg}$ & $\begin{array}{l}\text { Added mass in the sway y-axis of boat [4, } \\
5] \text {, and } p=1000 \mathrm{~kg} / \mathrm{m}^{3} \text { density of water }\end{array}$ \\
\hline$I$ & $\begin{array}{l}\left(m\left(W^{2}+L^{2}\right) / 12\right) \\
\mathrm{kg} \mathrm{m}^{2}\end{array}$ & $\begin{array}{l}\text { Mass moment of inertia of boat about } \\
\text { mass centre in yaw } \mathrm{z} \text {-axis }\end{array}$ \\
\hline$I_{\mathrm{zz}}$ & $\begin{array}{l}\left(0.1 m W^{2}+p \pi D^{2} L^{3}\right) \\
/ 2 / 12 \mathrm{~kg} \mathrm{~m}^{2}\end{array}$ & $\begin{array}{l}\text { Linear coefficient of added mass moment } \\
\text { of inertia in } \mathrm{z} \text {-axis }[4,5]\end{array}$ \\
\hline$d_{1}$ & 14 & $\begin{array}{l}\text { Hydrodynamic damping coefficient of } \\
\text { surge } x \text {-axis [4] }\end{array}$ \\
\hline$d_{2}$ & 80 & $\begin{array}{l}\text { Hydrodynamic damping coefficient of } \\
\text { sway y-axis }\end{array}$ \\
\hline$d_{3}$ & 5 & $\begin{array}{l}\text { Hydrodynamic damping coefficient of } \\
\text { yaw z-axis }\end{array}$ \\
\hline$a_{1}$ & 1 & $\begin{array}{l}\text { Exponential damping coefficient of surge } \\
\mathrm{x} \text {-axis [4] }\end{array}$ \\
\hline$a_{2}$ & 1 & $\begin{array}{l}\text { Exponential damping coefficient of sway } \\
y \text {-axis }\end{array}$ \\
\hline$a_{3}$ & 1 & $\begin{array}{l}\text { Exponential damping coefficient of yaw } \\
\text { z-axis }\end{array}$ \\
\hline
\end{tabular}
budget catamanran test boat built with two PVC ducts, acrylic blocks and varnished Medium-Density Fabreboards (MDFs). The kinematic and kinetic varibales are listed in Table II. The range for thrust angle $b$ is determined by the servo used and thrust force $F$ by the brushless motor (Appendix A Fig. A1).

TABLE I. Boat parameters

The values for nonlinear hydrodynamic damping coefficients $\left(d_{1}, d_{2}, d_{3}, a_{1}, a_{2}, a_{3}\right)$ in equations ( 4 to 5$)$ are estimated based on the force-velocity relationships in each of the surge, sway and yaw axis obtained by Muske et al. [4] on a half-metre long model boat. These values provide a starting point for the designers to evaluate their simulation using experimental techniques described in [4]. These coefficients depend largely on the hull form, size and speed of the boat. Units of $d_{\mathrm{i}}$ and $a_{\mathrm{i}}$ (i $=1,2,3)$ are SI standard to give rise to $\mathrm{N}$ for $\left(d_{\mathrm{i}} \cdot \operatorname{sign}\left(x^{\prime}\right) \cdot\left|x^{\prime}\right|^{a_{\mathrm{i}}}\right)$.

The uniform disturbance force $F_{\mathrm{d}}$ is modelled as a sinusoidal time function of long-crested wave with point of action at the mass centre of the boat [1].

$$
F_{\mathrm{d}}=A \sin \left(w_{\mathrm{d}} t\right)+B
$$

Where $A$ is the amplitude of the disturbance force $(\mathrm{N}), w_{\mathrm{d}}$ is the oscillatory frequency of the disturbance force ( $\mathrm{rad}), B$ is the 
bias - the constant compoennet of force that applies $(\mathrm{N}), t$ is time (s). Usually $A<B$.

The boat heading $z$ and disturbance force angle $k$ are both measured relative to the $+\mathrm{X}$ of the global inertial frame, while the thurst angle $b$ is measured relative to the $+\mathrm{x}$ of local fixedbody frame (see Fig. 2 and 3).

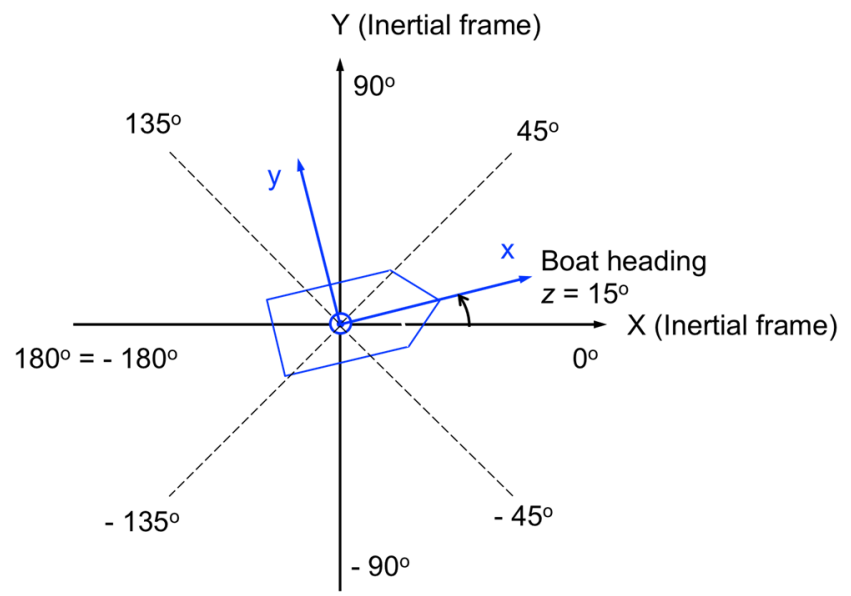

Fig. 3. Angluar relations defining the four quadrants and boundaries in the global inertial frame. Boat heading $z$ and disturbance angle $k$ are always measured relative to the $+X$ inertial frame.

The thrust force $F$ and thrust angle $b$ are determined using a logic control to follow the heading angle demand for the position of the next waypoint. 'Track-keeping' is not implemented in the present study as the primary interest is to identify boat control dynamics at close proximity.

The dynamic relations and control algorithms are simulated using MATLAB-Simulink ${ }^{\circledR}$ (2016a). The three equations of motion $(4-6)$ are integrated using a Runge-Kutta fourth order integrator.

TABLE II. Kinematic and kinetic variables

\begin{tabular}{|c|c|c|}
\hline & Unit & Defintion \\
\hline$x, x^{\prime}, x^{\prime \prime}$ & $\mathrm{m}, \mathrm{m} / \mathrm{s}, \mathrm{m} / \mathrm{s}^{2}$ & $\begin{array}{l}\text { Current boat position, velocity, acceleration } \\
\text { in local fixed-body } \mathrm{x} \text {-axis (surge) }\end{array}$ \\
\hline$y, y^{\prime}, y^{\prime \prime}$ & $\mathrm{m}, \mathrm{m} / \mathrm{s}, \mathrm{m} / \mathrm{s}^{2}$ & $\begin{array}{l}\text { Current boat position, velocity, acceleration } \\
\text { in local fixed-body y-axis (sway) }\end{array}$ \\
\hline$z$ & $\mathrm{rad}$ & $\begin{array}{l}\text { Current boat heading relative to }+X \text { of } \\
\text { inertial frame }[-\pi, \pi] \text {, yaw }\end{array}$ \\
\hline$z^{\prime}, z^{\prime \prime}$ & $\mathrm{rad} / \mathrm{s}, \mathrm{rad} / \mathrm{s}^{2}$ & $\begin{array}{l}\text { Boat heading angular velocity and angular } \\
\text { acceleration }\end{array}$ \\
\hline$b_{\max }$ & $0.47 \pi \mathrm{rad}$ & Maximum thrust angle \\
\hline$b$ & $\mathrm{rad}$ & $\begin{array}{l}\text { Thrust force angle relative to current boat } \\
\text { heading }+\mathrm{x} \text { of local fixed-body frame }\left[-b_{\max } \text {, }\right. \\
\left.b_{\max }\right]\end{array}$ \\
\hline$k$ & $\mathrm{rad}$ & $\begin{array}{l}\text { Disturbance force angle relative to }+\mathrm{X} \text { of } \\
\text { inertial frame }[-\pi, \pi]\end{array}$ \\
\hline$F_{\max }$ & $20 \mathrm{~N}$ & Maximum thrust force \\
\hline$F$ & $\mathrm{~N}$ & Thrust force $\left[0, F_{\max }\right]$ \\
\hline$F_{\mathrm{d}}$ & $\mathrm{N}$ & $\begin{array}{l}\text { Disturbance force acting at mass centre } \\
\text { assuming long-crested wave [1] }\end{array}$ \\
\hline
\end{tabular}

\section{B. Control logic}

A pseudo code depicts five steps to find thrust force $F$ and thrust angle $b$ in order to reach the next waypoint. See Appendix B for the controller implemented using MATLAB function ' $\operatorname{nav}($.$) ' as part of the Simulink model with an$ overview in Fig. 4.

1) Obtain next waypoint $\left(X_{\mathrm{w}}, Y_{\mathrm{w}}\right)$, current position $(X, Y)$, heading $(z)$, max thrust force $F_{\max }$ and thrust angle $b_{\max }$.

2) Compute demand heading:

$h d=\operatorname{atan} 2\left(\left(Y_{\mathrm{w}}-Y\right) /\left(X_{\mathrm{w}}-X\right)\right)$, where 'atan2(.)' returns four-quadrant inverse tangent in the range $[-\pi, \pi]$;

current distance to next waypoint:

$d s t=\sqrt{ }\left(\left(Y_{\mathrm{w}}-Y\right)^{2}+\left(X_{\mathrm{w}}-X\right)^{2}\right)$;

heading difference between demand and current heading:

dhd $=h d-z$.

3) Ensure heading difference $d h d$ is in range $[-\pi, \pi]$.

$$
\begin{aligned}
& \text { if } d h d<-\pi: \\
& \quad d h d=2 \pi+h d-z ; \\
& \text { elseif } d h d>\pi: \\
& \quad d h d=-2 \pi+h d-z .
\end{aligned}
$$

4) Check if distance to next waypoint $d s t$ is less than $2.5 \mathrm{~m}$.

if $d s t<=2.5$ : else: stop $(F=0, b=0)$ or update waypoint; go to step 5).

5) Determine the direction to turn.

\# for small turning angle

if $($ dhd $>-\pi / 2)$ and $($ dhd $<\pi / 2)$ :

$b=b_{\max } d h d /(\pi / 2) \quad \#$ proportional to $d h d ;$

$F=F_{\max } \# \max$ thrust;

\# for large turning angles

elseif $(($ dhd $>=-\pi)$ and $($ dhd $<=-\pi / 2))$ or

$(($ dhd $>=\pi / 2)$ and $($ dhd $<=\pi))$ :

$b=b_{\max }(\operatorname{sign}(d h d)) \quad \# \max$ thrust angle to turn.

if $d s t<=10$ :

$F=\left(F_{\max } / 3\right)(d s t / 10) \quad \#$ proportionally reduced thrust when close to waypoint;

else:

$F=F_{\max } / 2 \quad \#$ halved thrust for large turning angle.

At each integration step, the above control algorithm updates its current boat (mass centre) position in the global inertial frame $(X, Y)$ in step 1$)$, and computes the heading difference $d h d$ and distance to the waypoint $d s t$ in step 2). Step 3 ) ensures the heading difference $d h d$ to be in the range $[-\pi, \pi]$, so as to prevent swirling. Step 4) sets the tolerance for reaching a waypoint. A distance of $2.5 \mathrm{~m}$ is considered reasonably accurate for a boat of one metre long and half a metre wide.

Step 5) is the main iterative step of boat control before reaching the waypoint tolerance. First, the turns are classified 
as 'small turn' with heading difference $d h d$ in the range $[-\pi / 2$, $\pi / 2]$ and 'large turn' with heading difference $d h d$ in $[-\pi,-\pi / 2]$ or $[\pi / 2, \pi]$. In the case of a small turn (less than $\pi / 2$ ), thrust angle $b$ is proportional to heading difference $d h d$ with a fixed gain of $b_{\max } /(\pi / 2)$; thrust force $F$ is set to maximum $F_{\max }$. In the case of a large turn (between $\pi / 2$ and $\pi$ ), thrust angle $b$ is set to maximum $b_{\max }$. But the thrust force $F$ is adjusted according to current distance to waypoint $d s t$. If this distance is less than 10 $\mathrm{m}$, the thrust force $F$ is reduced in proportion to $d s t$ by multiplying $\left(F_{\max } / 3\right)$ with $(d s t / 10)$. If the distance is greater than $10 \mathrm{~m}$, the thrust force is limited to a half of the maximum. To experience, the reduced thrust force provides a better chance for the boat to reach the waypoint at large turns between $\pi / 2$ and $\pi$ at close proximity. As a rule of thumb, a distance from 10 to $20 \mathrm{~m}$ seems to give a good heading control based on the specification of the current boat: $F_{\max }, b_{\max }$, mass moment of inertia, length, width, hydrodynamic damping coefficients to turn. These factors affect boat forward speed and maximum turning moment that dominate the behaviour of the boat when approaching the $2.5-\mathrm{m}$ radius tolerance of the waypoint. The ultimate behaviour would inevitably be influenced and complicated by the direction $(k)$ and magnitude of the disturbance force $F_{\mathrm{d}}$.

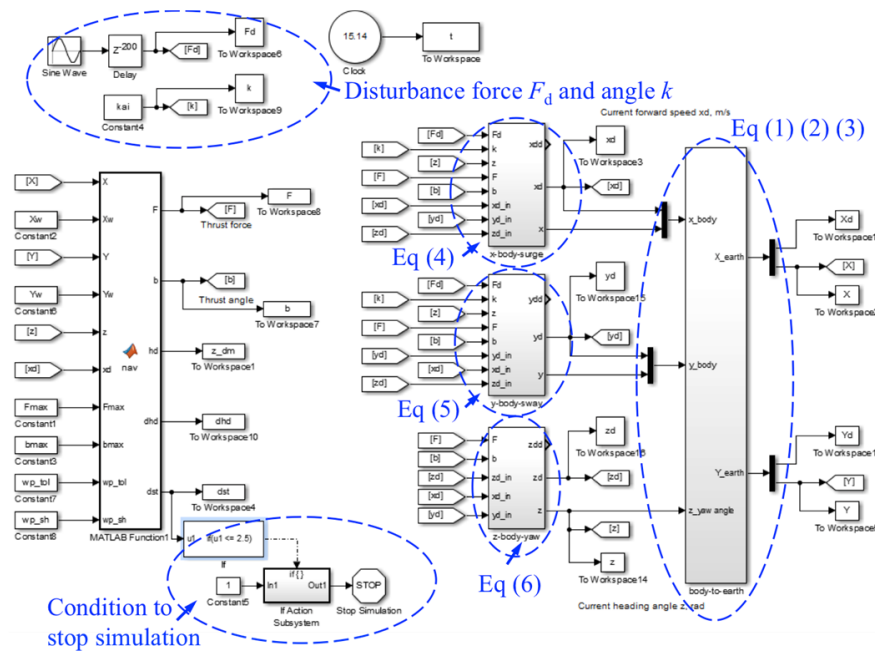

Fig. 4: Navigation and control module (left 'nav'), and boat dynamics module (right cluster) with equations (1) to (6) implemented in Simulink where $x d=x$ ' and $x d d=x$ " for example.

\section{RESULTS}

During the simulation the boat starts to move from rest at the global inerital frame origin $(0,0)$ to a waypoint $\left(X_{\mathrm{w}}, Y_{\mathrm{w}}\right)$ also defined in the global frame. The simulation is terminated once the boat reaches the $2.5-\mathrm{m}$ radius tolerance of the waypoint. The resutls are divided into conditions with and without disturbance force. If not mentioned, the 'benchmark' configuration is used. The 'benchmark' is based on parameters listed in Tables I and II, and two threshold distances to waypoint at 2.5 and $10 \mathrm{~m}$ (see Section II B).

\section{A. Still water simulation}

The minimum radius of turn trial set the target waypoint to $(-1.5,0)$ with a radius tolerance of $1 \mathrm{~m}$. Effectively the boat is tasked to travel from rest at an initial heading angle at $+\mathrm{X}$ or $0^{\circ}$ to reach a circle of $1-\mathrm{m}$ tolerance radius centred at $(-1.5,0)$. By varying the maximum thrust force from 5 to $80 \mathrm{~N}$, Fig 5 shows the miminum radius of turn is around $0.2 \mathrm{~m}$. A $20 \mathrm{~N}$ thrust capacity provide a compromise between turning range and forward speed. This radius is only a fraction of the boat length $(1 \mathrm{~m})$ indicating the tightest turn achievable in still water. Apart from this trial, the rest of the simulation adopted a $2.5-\mathrm{m}$ radius for waypoint distance tolerance.

The trajectories presented in Fig. 6 show that the simulated boat is able to reach and converge to waypoints in all directions of $[-\pi, \pi]$ starting from rest at $(0,0)$ with initial heading to $+\mathrm{X}$. The maximum forward 'cruise' speed is found to be $1.4 \mathrm{~m} / \mathrm{s}$, and the times and distances to reach that speed at different angles of heading are listed in Table III.

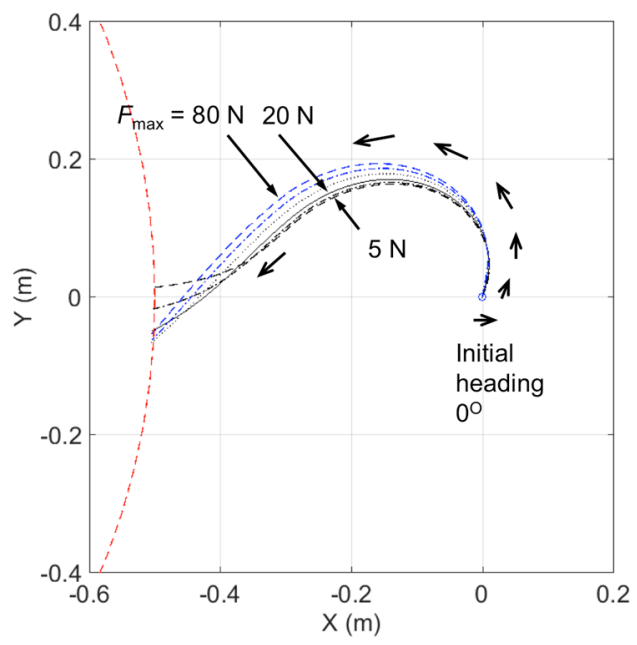

Fig. 5. Comparison of boat trajectories at minimum turning radius (thrust angle $\left.b=b_{\max }=0.47 \pi\right)$ at six settings of maximum thrust force $F_{\max }(5,10,20,40$, 60 , and $80 \mathrm{~N})$ showing starting $(0,0)$ and stopping target waypoint $(-1.5,0)$ in still water. The arch shows part of the target tolerance circle with a radius of 1 $\mathrm{m}$. The boat is initially at rest and heading to $+\mathrm{X}$ or $0^{\circ}$.

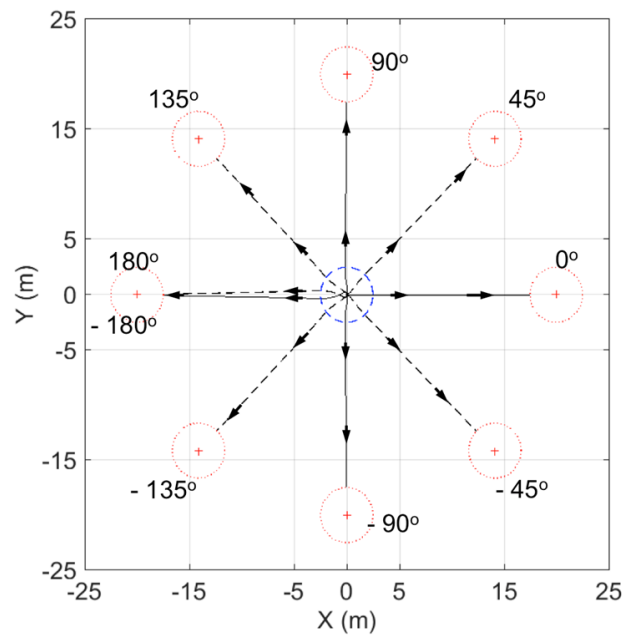

Fig. 6. Nine trajectories (solid and dashed lines) simulated with 9 initial demand heading angsles towards 8 waypoints from origin $(0,0)$ with an initial heading of $0^{\circ}$. The dotted circles show the $2.5-\mathrm{m}$ radius to waypoints marked by a ' + '. 
TABLE III. Time and distance to reach the maximum forward speed of $1.4 \mathrm{~m} / \mathrm{s}$ at different angles by setting the waypoints, for example $(0,-20)$ for an angle of $-90^{\circ}$ (see Fig. 3 and 6).

\begin{tabular}{ccc}
\hline Heading angle & Time (s) & Distance $(\mathrm{m})$ \\
\hline $0^{\circ}$ & 2.4 & 2.7 \\
$45^{\circ}$ or $-45^{\circ}$ & 2.6 & 2.8 \\
$90^{\circ}$ or $-90^{\circ}$ & 3.0 & 3.0 \\
$135^{\circ}$ or $-135^{\circ}$ & 4.1 & 3.1 \\
$180^{\circ}$ or $-180^{\circ}$ & 5.1 & 3.2 \\
\hline
\end{tabular}

\section{B. Disturbance force simulation}

The results illustrate simulated effects of disturbance force $F_{\mathrm{d}}$ and its angle $k$ on the boat trajectories, while heading to waypoint $(20,0)$ positioned at $0^{\circ}$. The battery of trials is based on parameters defined in Equation (7) shown in Table IV. $k$ is the angle of disturbance force measured from $+\mathrm{X}\left(0^{\circ}\right)$ acting on the mass centre of the boat. $B$ is the bias of disturbance force, and $A$ is the oscillatory amplitude of distrubance force. The disturbance force oscillation frequency $w_{\mathrm{d}}=0.4 \pi \mathrm{rad} / \mathrm{s}$ (or 0.2 $\mathrm{Hz}$ ) and oscillatory anmolitude $A=5 \mathrm{~N}$ are fixed in the simulation. According to Fossen [1], the dominant wave frequency is in the range $[0.01,0.2] \mathrm{Hz}$.

TABLE IV. Disturbance trial battery

\begin{tabular}{cccc}
\hline Trial no. & Heading angle & $k(\mathrm{rad})$ & $B(\mathrm{~N})$ \\
\hline D1 & $0^{\circ}$ & $\pi / 4$ & 10 \\
D2 & & & 20 \\
D3 & & $\pi / 2$ & 30 \\
D4 & & 10 \\
D5 & & 20 \\
D6 & $\pi 3 / 4$ & 30 \\
D7 & & 10 \\
D8 & & 20 \\
D9 & & 30 \\
\hline
\end{tabular}

The layoout of Fig. 7 is used to check key variables and behaviours of the boat. Disturbance force introduced in D1 (Fig. 7) is considered moderate. More severe conditions D3, D6, D8 and D9 are presented in subsequent Fig. 8 to 11. (a)

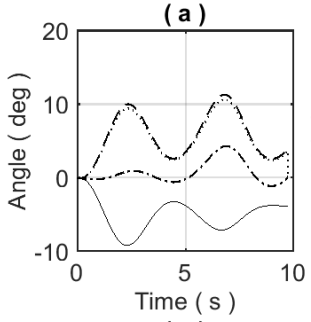

(c)

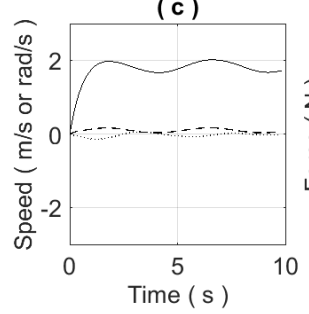

(b)

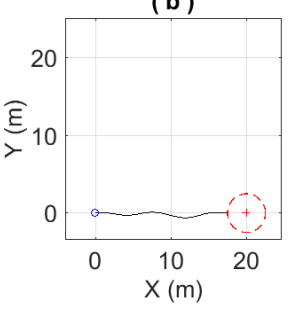

(d)

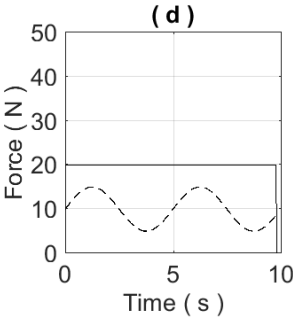

Fig. 7. Trial D1 $(k=\pi / 4, B=10 \mathrm{~N})$ : (a) Current boat heading ( $\quad$ ), demand heading (.$\left._{-}\right)$, heading difference $\left({ }_{-}{ }_{-}\right)$and closely thrust angle $(\ldots)$; (b) Boat trajectory starting from $(0,0)$ to the waypoint at $(20,0)$ with 2.5 -m radius; (c) Surge speed $(\ldots, \mathrm{m} / \mathrm{s})$, sway speed $\left({ }_{-}, \mathrm{m} / \mathrm{s}\right)$ and yaw angular speed $(\ldots, \mathrm{rad} / \mathrm{s})$; (d) Thrust force (_ $~)$ and disturbance force $\left({ }_{-}{ }_{-}\right)$.
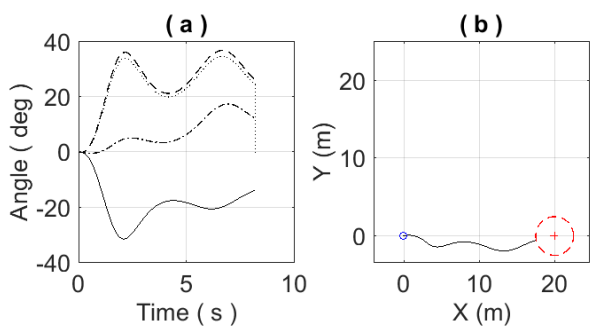

(c)
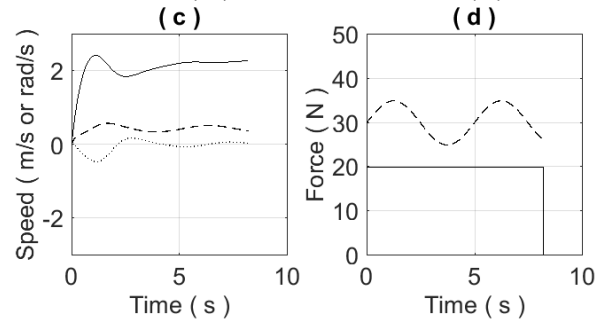

Fig. 8. Trial D3 $(k=\pi / 4, B=30 \mathrm{~N})$
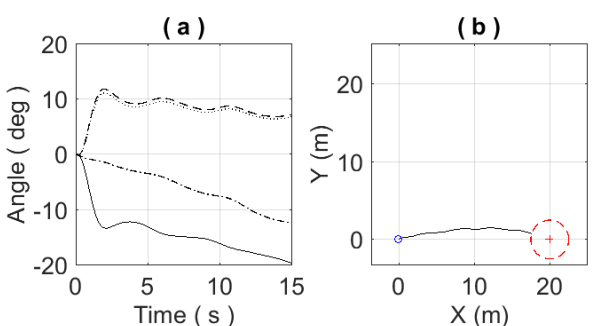

(c)

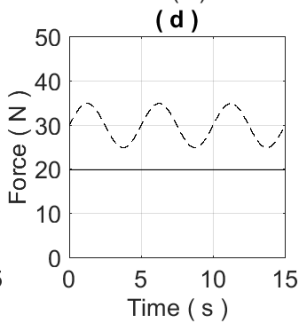

Fig. 9. Trial D6 $(k=\pi / 2, B=30 \mathrm{~N})$
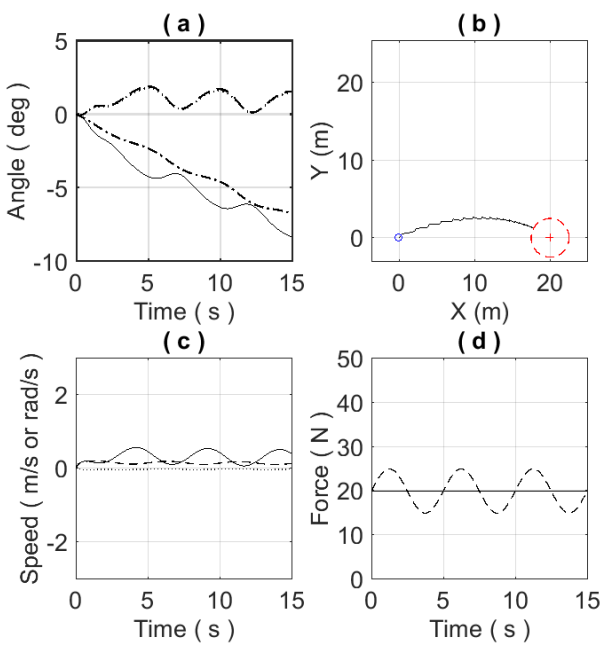

Fig. 10. Trial D8 $(k=\pi 3 / 4, B=20 \mathrm{~N})$ 

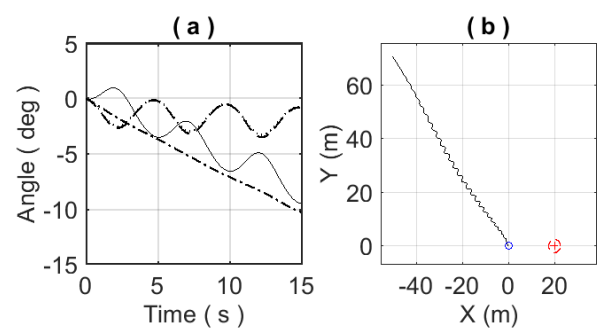

(c)
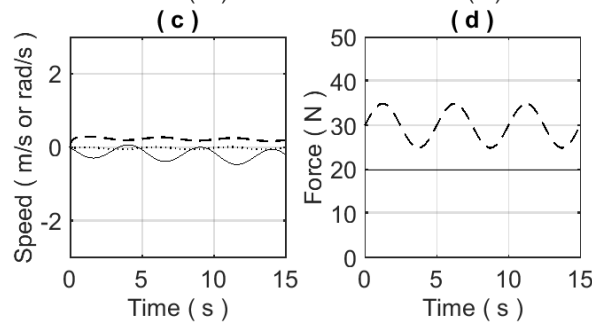

Fig. 11: Trial D9 $(k=\pi 3 / 4, B=30 \mathrm{~N})$

At disturbance force angles of $\pi / 4$ and $\pi / 2$, the boat is able to converge and reach the waypoint at the highest steady disturbance force of $B=30 \mathrm{~N}$ (Fig. 8: Trial D3 and Fig. 9: Trial D6). As disturbance force angle increased to $\pi 3 / 4$, the boat struggled (Fig. 10, $B=20 \mathrm{~N}$ ), failed at $B=30 \mathrm{~N}$ (Fig. 11).

\section{DISCUSSION}

\section{A. Assumptions and uncertainties of simulation}

Very often hydrodynamic parameters are not available until a prototype boat is designed, constructed and tested. This throws doubts on the simulated results. The presented simulation intends to provide the first steps towards a more realistic representation of the boat dynamic behaviour [6]. However, the damping coefficients, added masses, and disturbance force function adopted in the present study are speculative with the aim to illustrate the simplified modelling approach rather than any quantitative judgement.

From a mechanical design point of view, the simulation of a three degrees of freedom surge-sway-yaw model could inform the prototyping process in a few steps.

1) Determine payload to be carried by the boat, and therefore the buoyancy and hull size required.

2) Derive hydrodynamic added mass coeffients in the surge-sway-yaw axes $\left(m_{\mathrm{xx}}, m_{\mathrm{yy}}, I_{\mathrm{zz}}\right)$. Refer to Chapter 4 of [5], Chapter 6 and 7 of [1], and Section 3 of [4].

3) Derive hydrodynamic damping coefficients in the surge-sway-yaw axes $\left(d_{1}, d_{2}, d_{3}, a_{1}, a_{2}, a_{3}\right)$. Refer to Chapter 7 of [1], and [4] for a experimental approach using a prototype. The prototype could be replica of simlar geometries and sizes.

4) Take some measurement of the wind and current forces around the waterway, where the boat will be operated using a Newton meter in a similar setup to the one outlined in Fig. A1. This estimates a range of the disturbance force $F_{\mathrm{d}}$. Some practical techniques of estimation are mentioned in [3] by scaling a model.
5) Idenitfy requirements for thrust force $F_{\max }$ and range of thrust angle $b_{\max }$ considering turning radius and disturbance using the simulation framework.

6) Develop control logics and distributed actuators (thrusters) in the numeric simulation to inform design options with improved seakeeping and manoeuverability.

\section{B. Actuator layout driven controller design}

In the numeric simualtion, it is possible to evaluate the different control strategies according to actuator layout.

Three possible actuator layouts are proposed in Fig. 12 which could be implemented in the numeric simulation by applying different equations of motion. The catamaran form has been favoured by the USV community due to its stability, payload capacity, and serviceability [7].

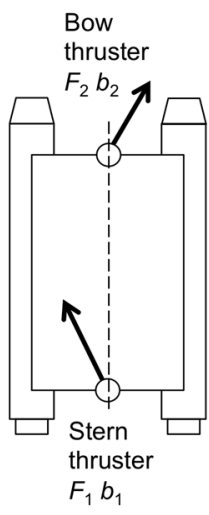

(a)

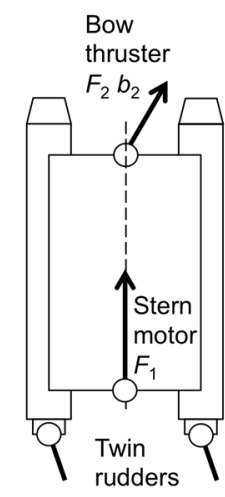

(b)

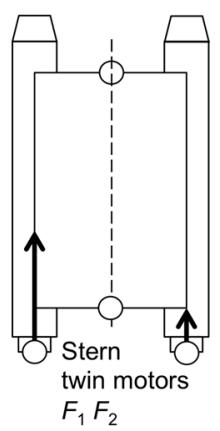

(c)
Fig. 12. Possible layouts of actuators for a autonomous surface vehicle. $F$ is thrust force usually provided by an electric motor, and $b$ is the thrust or rudder angle relative to the boat fixed referenec frame.

Design (a) provides the most flexible manoeuverability and attitude control - ideal for tasks involving self-docking, narrow waterways, and moving target tracking. The boat is able to 'turn on spot' with a 'couple' to provide maximum turning moment. Were one of the two thrusters down, the second could still make good.

Design (b) was adopted in the original Pytheas project at Portsmouth. The bow thruster was added to compensate large turning angles $\left(>90^{\circ}\right)$ where the stern motor-twin rudder combination proved inadequate. At small turning angles of $\left[0^{\circ}\right.$, $\left.90^{\circ}\right]$ or $\left[90^{\circ}, 0^{\circ}\right]$, the controller was set up to only engage rudder servos to turn with bow thruster fixed at $0^{\circ}$ and motor on full power, and stern motor on full power too. At large turning angles of $\left[90^{\circ}, 180^{\circ}\right]$ or $\left[-180^{\circ},-90^{\circ}\right]$, the stern motor is off, the rudders are at $0^{\circ}$ (straightened), and the bow thruster is at full power with its servo at maximum turning angle. In addition to the effective turning ability, an unexpected benefit of this design was to have the twin rudders acting as dampers to prevent oscillatory wave disturbances at sea [1], especially at turns. It was not able to turn at spot and therefore tasks such as self-docking would rely on a robust control logic. The number of actuators provided adequate system redundancy.

Design (c) has been a popular choice for a number of USV projects, e.g. [3]. The difference in thrust force between the two stern motors provides the turning moment. The design is 
the simpliest. If one motor is down, the other can still make its way home with suitable control logic. However, some oscillation may occur during a turn if control logic and filters in deed are not carefully calibrated with disturbance from wave. A different version of this layout used servo controlled motors at the two stern locations to provide enhanced manoeuverability.

\section{CONCLUSIONS}

The simulation framework allows designers to ask what-if questions before setting out to construct their prototypes. Although a definitive answer to 'how far away is it from the high sea?' seems unreachable, the work at least points us towards the direction of what needs to be done. While every vessel is different in its own hydrodynamic form in the high sea, their control logic should be informed right from the mechanical design process.

\section{Appendix A Hardware of Pytheas project at Portsmouth}

\section{Electronics}

- Raspberry Pi 2 Model B (5V 500mAh)

- LiPo Batteries: Zippy 7.4v 6000mAh LiPo 2S 50C battery

- Battery splitter between battery and ECSs, Voltage regulators

- Voltage regulator LM2596 DC-DC adjustable PSU (5V 3A)

- WiFi USB Dongle: Vilros Raspberry Pi Compatible (100mAh)

- Adafruit USB Ultimate GPS with External Antenna Breakout (20mAh)

- Inertial Measurement Unit (IMU): BerryIMU Ozzmaker.com $(3.3 \mathrm{~V})$

- Electronic Speed controllers (ECSs): Hobby King Brushless (7.4V $\sim 9 \mathrm{Ah})$

- Brushless Motors: NTM Prop Drive 28-30A 750kv 140w

- Servos: Towerpro MG996R Servo 10kg / 0.20sec / 55g (1000mAh)

- Pi Camera (250mAh)

\section{Construction material}

- Acrylic plates: positioning of motors

- Plastic solid ducting and caps: twin hulls

- MDF plates: top and bottom decks, and cross beams

- Metal push rods: between servos and motors

- Bolts, nuts, straps, glue, vanish

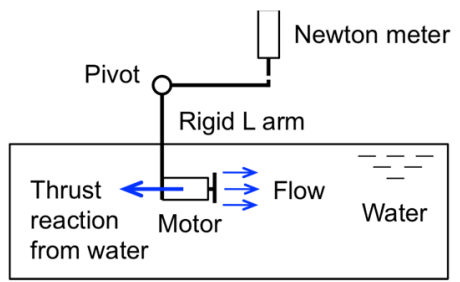

Fig. A1. Test bench to find thrust force produced by the brushless motor. Each motor has a maximum thrust of $10 \mathrm{~N}$ and a pair is used to produce $20 \mathrm{~N}$.

\section{Appendix B Control logic}

The navigation controller to find thrust force $F$ and thrust angle $b$ in order to reach the next waypoint implemented in a MATLAB function 'nav' in the Simulink model (Fig. 4).

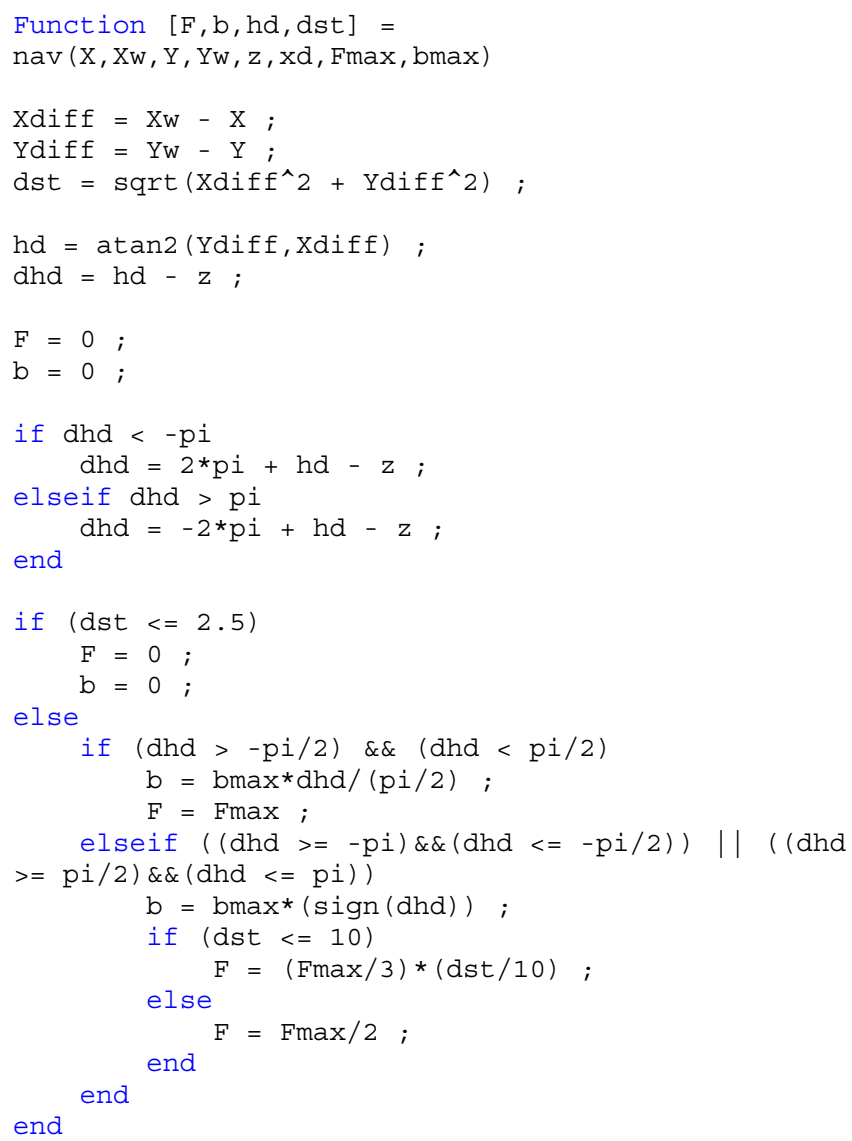




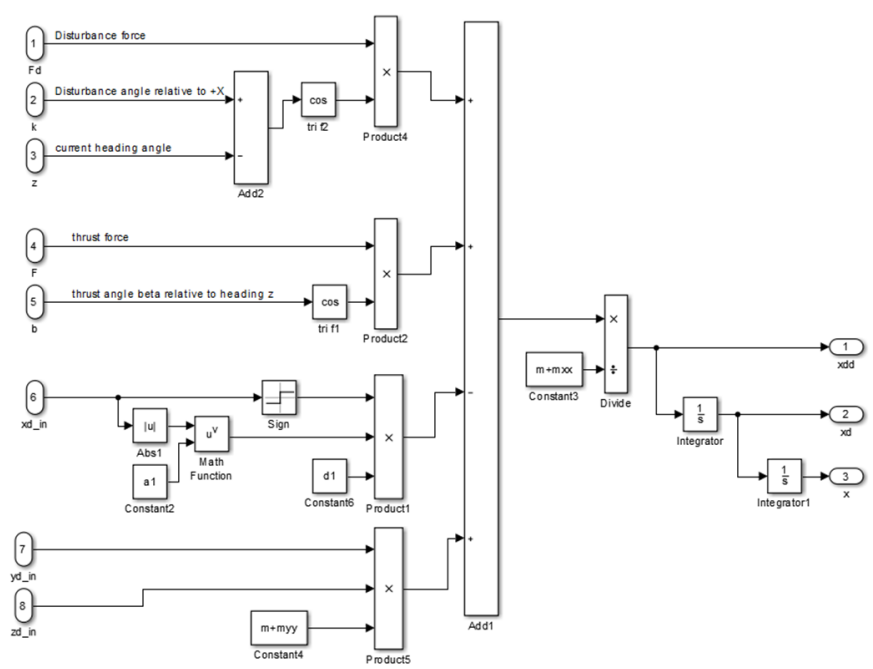

Fig. B1. Simulink model for Equation (4)

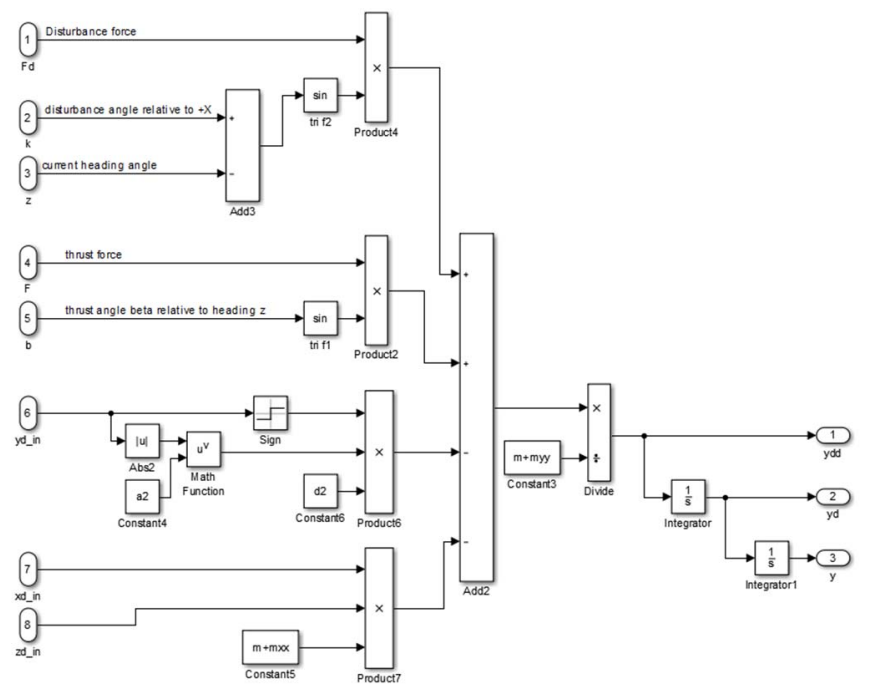

Fig. B2. Simulink model for Equation (5)

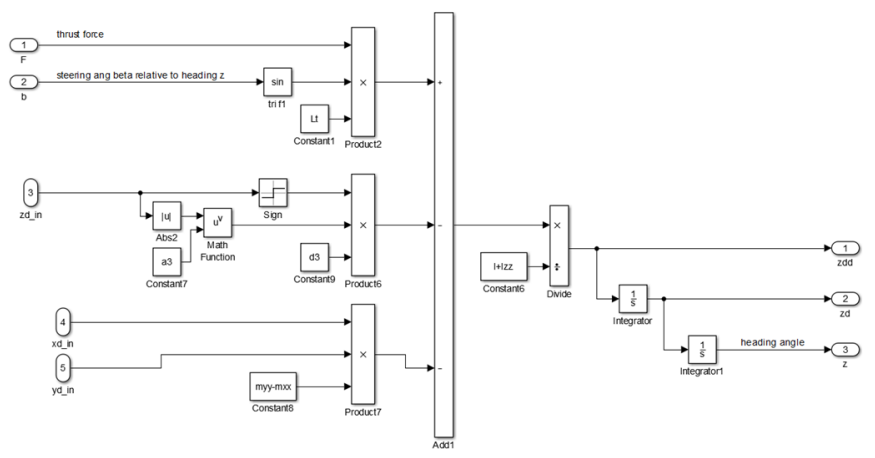

Fig. B3. Simulink model for Equation (6)

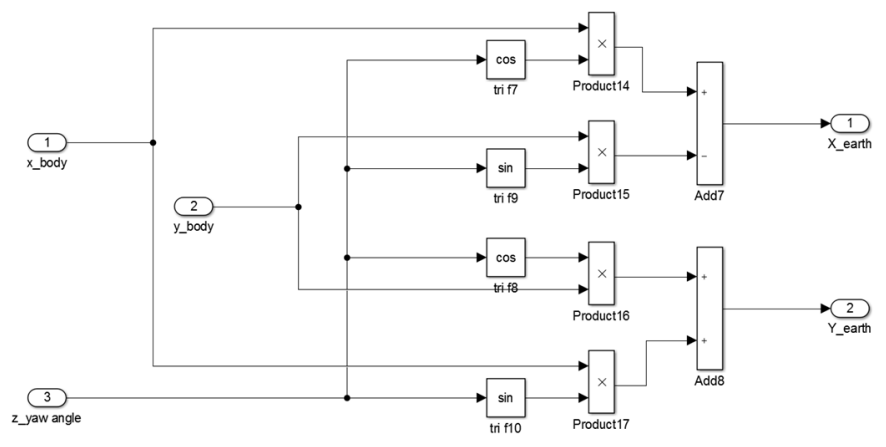

Fig. B4. Simulink model for equations (1), (2), and (3)

\section{REFERENCES}

[1] T.I. Fossen, Handbook of marine craft hydrodynamics and motion control. Chichester, UK: John Wiley \& Sons Ltd., 2011

[2] S. Campbell, W. Naeem, GW. Irwin, A review on improving the autonomy of unmanned surface vehicles through intelligent collision avoidance manoeuvres. Annual Reviews in Control, 36, pp. 267-283, 2012

[3] F. Mahini, L. DiWilliams, K. Burke, H. Ashrafiuon, An experimental setup for autonomous operation of surface vessels in rough seas. Robotica, 31, pp. 703-715, 2013

[4] K.R. Muske, H. Ashrafiuon, G. Haas, R. McCloskey, T. Flynn, Identification of a control oriented nonlinear dynamic USV model, American Control Conference, Seattle, WA, pp. 562-567, 2008

[5] J.N. Newman, Marine hydrodynamics. MIT Press, 1977

[6] P.G. Thomasson, C.A. Woolsey, Vehicle motion in currents. Journal of Oceanic Engineering, 38(2), pp. 226-242, 2013

[7] A. Martins, H. Ferreira, C. Almeida, H. Silva, J.M. Almeida, E. Silva, ROAZ and ROAZ II Autonomous Surface Vehicle Design and Implementation. International Lifesaving Congress, La Coruna, Spain, 2007 